\title{
Research Paper: Test-Retest Reliability of Surface Electromyographic Measurements in Athletes With Anterior Cruciate Ligament Reconstruction: A Voluntary Response Index Analysis
}

Elie Hajouj ${ }^{1}$ (D, Mohammad Reza Hadian ${ }^{1,2^{\star}}$ (D), Seyed Mohsen Mir ${ }^{1}$ (D), Saeed Talebian' ${ }^{1}$, Farzin Halabchi ${ }^{3}$ (D), Salah Ghazi ${ }^{1}$ (iD)

1. Department of Physiotherapy, School of Rehabilitation, Tehran University of Medical Sciences, Tehran, Iran.

2. Brain and Spinal Cord Injury Research Center, Institute of Neuroscience., Tehran University of Medical Sciences, Tehran, Iran.

3. Department of Sports and Exercise Medicine, Tehran University of Medical Sciences, Tehran, Iran.

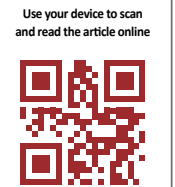

Citation: Hajouj E, Hadian MR, Mir SM, Talebian S, Halabchi F, Ghazi S. Test-Retest Reliability of Surface Electromyographic Measurements in Athletes With Anterior Cruciate Ligament Reconstruction: A Voluntary Response Index Analysis. Journal of Modern Rehabilitation. 2020; 15(1):1-6. http://dx.doi.org/10.32598/JMR.15.1.1

http://dx.doi.org/10.32598/JMR.15.1.1

Article info:

Received: 27 Jul 2020

Accepted: 31 Aug 2020

Available Online: 01 Jan 2021

Keywords:

Test-retest reliability,

Voluntary response index,

Anterior cruciate ligament reconstruction, Motor control, Rehabilitation

\section{ABSTRACT}

Introduction: Voluntary Response Index (VRI) is used as a measure of motor control to study abnormalities of voluntary movements. This study aimed to evaluate the reliability of voluntary response index analysis in subjects with Anterior Cruciate Ligament Reconstruction (ACLR).

Materials and Methods: Using surface electromyography, the VRI components of both groups of 15 ACLR and 15 healthy controls were assessed during the functional task (sit-standsit). The outcome variables of VRI included the magnitude and similarity index.

Results: In sit to stand phase, high reliability was found (ICC $=0.80-0.89$ ) for the magnitude and similarity index in both groups. In the standing phase, high to very high reliability was found for the magnitude and similarity index in both groups $(\mathrm{ICC}=0.75-0.91)$. In stand to sit phase, high to very high reliability was found $(\mathrm{ICC}=0.78-0.92)$ for the magnitude and similarity index in both groups.

Conclusion: Surface electromyographic measurements of VRI analysis demonstrated high to very high reliability in athletes with ACLR during the functional task (sit-stand-sit). The results of the current study showed that the VRI analysis in athletes after ACLR was a reliable method and can be used to evaluate motor control before and after ligament injury in these patients.

* Corresponding Author: 


\section{Introduction}

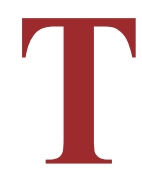

he Anterior Cruciate Ligament (ACL) is one of the most important ligaments that stabilizes the knee joint which contains mechanoreceptors [1]. Since, it contributes substantially to the joint stability and function [2], when seriously injured, the first choice for treatment for an athlete, most likely in orthopedics, is surgical reconstruction [3]. As we know, voluntary movements are produced by muscle activations and controlled by the Central Nervous System (CNS) [4]. A large number of mechanoreceptors and the nervous central links could be affected after ACL reconstruction (ACLR) causing changed movement patterns during various tasks and activities [5].

Voluntary Response Index (VRI) is a surface EMG technique with high sensitivity when used as a measure of motor control to study the abnormalities of voluntary movements [4, 6-11]. Instead of examining individual muscle activity, it would be more beneficial to examine the activation pattern of all muscles engaged in the entire prototype of a task. In practical application, the magnitude and the similarity activities of all muscles engaged in voluntary movement are studied as a whole rather than studying each muscle individually $[12,13]$.

Because of the amplitude variability across subjects, it is difficult to analyze the EMG signals that are recorded simultaneously from several muscles. The effect of variability across subjects is minimized by comparing the average patterns collected from healthy subjects by calculating the similarity index separately from the magnitude value. This method provides quantitative analysis and the elementary pattern recognition of voluntary motor control during different tasks [4]. The evidence about VRI is still under research. For example, Norouzi et al. used VRI to classify and distinguish subjects with ACL deficiency in a primitive stage of injury [13].

Generally, the test-retest reliability is used to measure the precision and consistency related to the repeatability of a variable to consider a specific method as a valid and reliable one.

The current study aimed to determine the test-retest reliability of quantitative measures of VRI analysis during the voluntary sit-stand-sit task. We hypothesized that the VRI method is reliable and therefore, it can be considered as an applicable technique during rehabilitation for future research in altered movement patterns studies in patients with ACLR.

\section{Materials and Methods}

\section{Study Participants}

Fifteen healthy and 15 ACLR subjects (i.e., control and case groups, respectively) voluntarily participated in this study. All subjects in the ACLR group had undergone reconstruction surgery with a similar technique, i.e., Semitendinosus and Gracilis Tendon (STG). The case group comprised amateur athletes from physical therapy clinics of rehabilitation faculty and the Sports Medicine Federation, and the control group comprised amateur athletes from the students at Tehran University of Medical Sciences. The inclusion criteria for the ACLR group were as follows: Athletes aged between 18 and 35 years, underwent a unilateral ACL reconstruction, no history or signs of injury in the contralateral knee, less than one month passed from injury to the surgery date, 8 weeks after ACLR, the Range of Movement (ROM) was greater than 100 degrees flexion and complete extension (0 degrees). Subjects were excluded if they had pain during tests or any complication preventing the data collection. The healthy subjects had no cardiopulmonary disease and neurological disorder or any history of musculoskeletal impairment in the last 3 months. Both groups were matched according to the demographic characteristic (Table 1).

\section{Test protocol}

The same examiner asked each subject to perform the functional task (sit-stand-sit). Each task was repeated 3 times in the test session with 30 seconds interval between the tasks. EMG activities of the 5 muscles of the knee, including the rectus femoris, vastus medialis, vastus lateralis, biceps femoris, and semitendinosus, were recorded during a sit-stand-sit task. The electrodes were placed based on the SENIAM's (Surface EMG for Non-Invasive Assessment of Muscles) recommendations (http://www. seniam.org/). All recordings were received through an 8-channel portable EMG system (DataLOG, Biometrics Ltd England). Preamplifier bipolar active electrodes with a fixed center-to-center interelectrode distance of $20 \mathrm{~mm}$, recording diameter of $10 \mathrm{~mm}$, built-in differential amplifier with a gain of 1000 , the input impedance of $1015 \Omega$, common-mode rejection ratio of $110 \mathrm{~dB}$ at $60 \mathrm{~Hz}$, and bandwidth of 20-450 Hz and ground electrodes located on the right wrist were attached to the skin.

The electrodes were numbered to match their entrances as follows: number 1 for rectus femoris, number 2 for vastus medialis, number 3 for vastus lateralis, number 4 for semitendinosus, and number 5 for biceps femoris. The subject was placed on a chair in the starting posi- 
Table 1. Demographic characteristic of the subjects in the experimental groups $(n=15)$

\begin{tabular}{ccc}
\hline & & Mean \pm SD \\
Variables & ACLR & Healthy \\
\cline { 2 - 3 } & & $23.7 \pm 2.66$ \\
Age $(\mathrm{y})$ & $173.1 \pm 4.55$ & $175.3 \pm 4.78$ \\
Height $(\mathrm{cm})$ & $76.7 \pm 4.87$ & $80.1 \pm 5.62$ \\
\hline
\end{tabular}

ACLR: Anterior Cruciate Ligament Reconstruction.

tion, with his back fully attached to the back of the chair, thus holding the hip joints at $90^{\circ}$ flexion. The knee joints were positioned at $90^{\circ}$. The electrodes were attached to the skin on the affected leg. Then, the subject was instructed to stand up in 5 seconds and holding this position for 10 seconds. After that, the subject was asked to sit in 5 seconds and holding this position for 10 seconds, and the cycle was repeated three times. The metronome was used to monitor phase rhythm during movement (i.e., a beep sounds every second). The subjects were asked to reach the point of complete standing on the 5 beeps, staying in this position for 10 beeps, return to sitting position on the 5 beeps, and staying in this position for 10 beeps. The subjects were asked to maintain a steady pace throughout the entire task.

\section{Data analysis}

The Magnitude (MAG) and the Similarity Index (SI) were the EMG outcome measures as they represent the two components of the VRI of the knee muscles during the sit-stand-sit task. VRI components express the amount of activity (MAG) and its distribution (SI) in comparison with the distribution of activity observed in healthy subjects when they are doing the same functional task [4]. The functional task (sit-stand-sit) was divided into three phases: phase 1, sit to stand; phase 2, stand; and phase 3 stand to sit.

To implement that, first, the Root Mean Square (RMS) was calculated for each muscle. In doing so, we got the Response Vector (RV) of that muscle. Afterward, the Prototype Response Vector (PRV) was calculated by placing the response vectors of the 5 muscles in Equation 1, which expresses the magnitude resultant of all muscles engaged in the functional task. Second, the SI was calculated using Equation 2. Equation 1 represents the magnitude of $\mathrm{n}$ muscles in a task and Equation 2 represents the SI of a group of muscles in a task, where the RVi is the RMS of each muscle, and the PRVi is the PRV of each muscle engaged in the task.
Equation 1: $R_{\text {noem }}=\frac{R^{1} R^{2} R^{3} \ldots R_{n}}{\sqrt{\sum_{i} R_{i}^{2}}}$

$\mathrm{Ri}=$ Response vector $(\mathrm{RMS})$ for each muscle

Equation 2: $S I=\frac{\sum_{i}(R V P R V)}{|R V||P R V|}$

Three trials of the functional task (sit-stand-sit) were used to calculate the reliability of the VRI. Considering the alpha level at 0.05 as statistically significant for all analyses.

\section{Statistical analysis}

The Intraclass Correlation Coefficient (ICC) was used to assess the reliability of the measures. A 95\% Confidence Interval (CI) was calculated for each ICC to bear the sampling distribution in mind. We calculated the relative intrasession reliabilities using a 1-way random model of the intraclass correlation coefficient [14]. To describe the degree of reliability, Munro's classification for reliability coefficients was used as follows: 0.00 0.25 , little, if any reliability; $0.26-0.49$, low reliability; 0.50-0.69, moderate reliability; 0.70-0.89, high reliability; and 0.90-1.00, very high reliability [15]. The Standard Error of Measurement (SEM) was used to assess absolute reliability which was calculated as the square root of the mean square error term obtained from the analysis of variance tables [16]. ICC and SEM for each phase of the task were calculated and presented in the related tables. SPSS v. 25 was used for all analyses.

\section{Results}

Mean \pm SD of the VRI components for the 3 trials in different phases of the functional task of the ACLR and healthy groups are shown in Table 2 . The ICC (95\% CI), SEM, and MDC are presented in Table 3. In general, the reliability of the VRI components, MAG, and SI ranged between high to very high reliability for both groups in all phases of the functional task. No significant differences were found between test and retest mean scores $(\mathrm{P}>0.05)$ of VRI components in the 3 phases of the func- 
Table 2. Descriptive data for the VRI components in 3 phases of the functional task (sit-stand-sit) in a sample of individuals with ACLR and healthy athletes $(\mathrm{n}=15)$

\begin{tabular}{|c|c|c|c|c|c|c|c|c|c|c|}
\hline \multirow{3}{*}{ Groups } & \multirow{3}{*}{ Variables } & \multicolumn{9}{|c|}{ Mean $\pm S D$} \\
\hline & & \multicolumn{3}{|c|}{ Sit to Stand } & \multicolumn{3}{|c|}{ Stand } & \multicolumn{3}{|c|}{ Stand to Sit } \\
\hline & & Test 1 & Test 2 & Test 3 & Test 1 & Test 2 & Test 3 & Test 1 & Test 2 & Test 3 \\
\hline \multirow{2}{*}{ ACLR } & $\mathrm{SI}$ & $0.23 \pm 0.07$ & $\begin{array}{c}0.23 \\
\pm 0.06\end{array}$ & $\begin{array}{c}0.21 \\
\pm 0.12\end{array}$ & $\begin{array}{c}0.40 \\
\pm 0.12\end{array}$ & $\begin{array}{c}0.45 \\
\pm 0.10\end{array}$ & $\begin{array}{c}0.39 \\
\pm 0.12\end{array}$ & $\begin{array}{c}0.23 \\
\pm 0.08\end{array}$ & $\begin{array}{c}0.19 \\
\pm 0.09\end{array}$ & $\begin{array}{c}0.18 \\
\pm 0.08\end{array}$ \\
\hline & MAG & $\begin{array}{l}33.91 \\
\pm 9.29\end{array}$ & $\begin{array}{c}31.90 \\
\pm 10.42\end{array}$ & $\begin{array}{c}31.98 \\
\pm 13.08\end{array}$ & $\begin{array}{c}8.98 \\
\pm 1.88\end{array}$ & $\begin{array}{l}10.08 \\
\pm 1.62\end{array}$ & $\begin{array}{c}9.10 \\
\pm 2.38\end{array}$ & $\begin{array}{c}34.96 \\
\pm 11.46\end{array}$ & $\begin{array}{c}32.57 \\
\pm 15.94\end{array}$ & $\begin{array}{c}32.02 \\
\pm 11.03\end{array}$ \\
\hline \multirow{2}{*}{ Healthy } & $\mathrm{SI}$ & $\begin{array}{c}0.43 \\
\pm 0.19\end{array}$ & $\begin{array}{c}0.44 \\
\pm 0.20\end{array}$ & $\begin{array}{c}0.56 \\
\pm 0.24\end{array}$ & $\begin{array}{c}0.27 \\
\pm 0.14\end{array}$ & $\begin{array}{c}0.30 \\
\pm 0.14\end{array}$ & $\begin{array}{c}0.37 \\
\pm 0.16\end{array}$ & $\begin{array}{c}0.52 \\
\pm 0.24\end{array}$ & $\begin{array}{c}0.51 \\
\pm 0.23\end{array}$ & $\begin{array}{c}0.52 \\
\pm 0.22\end{array}$ \\
\hline & MAG & $\begin{array}{l}54.87 \\
\pm 9.46\end{array}$ & $\begin{array}{l}52.56 \\
\pm 9.29\end{array}$ & $\begin{array}{c}53.62 \\
\pm 12.55\end{array}$ & $\begin{array}{c}30.82 \\
\pm 10.29\end{array}$ & $\begin{array}{c}24.74 \\
\pm 10.29\end{array}$ & $\begin{array}{c}26.35 \\
\pm 12.08\end{array}$ & $\begin{array}{c}53.33 \\
\pm 12.54\end{array}$ & $\begin{array}{c}44.71 \\
\pm 12.03\end{array}$ & $\begin{array}{r}44.63 \\
\pm 13.97\end{array}$ \\
\hline
\end{tabular}

JMR

ACLR: Anterior Cruciate Ligament Reconstruction; MAG: Magnitude $(\mu V)$; SI: Similarity Index

Table 3. Test re-test reliability of VRI components in 3 phases of the functional task (sit-stand-sit) in a sample of individuals with ACLR and healthy athletes

\begin{tabular}{|c|c|c|c|c|c|c|c|c|c|c|}
\hline \multirow{2}{*}{\multicolumn{2}{|c|}{ Variables }} & \multicolumn{3}{|c|}{ Sit to Stand } & \multicolumn{3}{|c|}{ Stand } & \multicolumn{3}{|c|}{ Stand to Sit } \\
\hline & & ICC (95\% CI) & SEM & MDC & ICC (95\% CI) & SEM & MDC & ICC (95\% CI) & SEM & MDC \\
\hline \multirow{2}{*}{ ACLR } & SI & 0.80 & 0.01 & 0.04 & 0.75 & 0.01 & 0.05 & 0.78 & 0.01 & 0.04 \\
\hline & MAG & 0.85 & 0.80 & 2.23 & 0.83 & 0.38 & 1.0 & 0.90 & 0.89 & 2.47 \\
\hline \multirow{2}{*}{ Healthy } & SI & 0.84 & 0.03 & 0.09 & 0.85 & 0.02 & 0.06 & 0.88 & 0.05 & 0.01 \\
\hline & MAG & 0.89 & 1.09 & 3.02 & 0.91 & 3.01 & 8.35 & 0.92 & 2.78 & 7.71 \\
\hline
\end{tabular}

JMR

ICC: Intraclass Correlation Coefficient; CI: Confidence Interval; SEM: Standard Error Of Measurement; MDC: Minimal Detectable Change; ACLR: Anterior Cruciate Ligament Reconstruction; SI: Similarity Index; MAG: Magnitude $(\mu V)$

tional task showing no systematic bias. The smaller SEM refers to the smaller error estimating between the tests.

In sit to stand phase, high reliability was found ( $\mathrm{ICC}=0.80-0.89$ ) for the MAG and SI in both groups. In the standing phase, high to very high reliability was found for the magnitude and similarity index in both groups ( $\mathrm{ICC}=0.75-0.91)$. In stand to sit position, high to very high reliability was found $(\mathrm{ICC}=0.78-0.92)$ for the MAG and SI in both groups.

\section{Discussion}

The current study examined the within-day test-retest reliability of surface electromyographic (sEMG) data analyzed through the Voluntary Response Index (VRI) method in subjects with and without ACLR during a functional task (sit-stand-sit). To the best of our knowledge, there is no study has yet addressed the reliability of sEMG data analysis by the Voluntary Response Index (VRI) method in athletes with ACLR in the sit-stand-sit task.

The results of the current study showed a high to very high reliability for the VRI method in all phases of the functional task. This implied that due to the small measurement errors compared with the variability between the subjects, the possibility of type 2 error was limited [14]. Furthermore, the ICC of MAG was higher than the ICC of SI within the ACLR and healthy groups in all phases of the functional task. Besides, the ICC of MAG and SI of the healthy subjects was higher than the MAG and SI of ACLR subjects; this may be due to more coordination between the studied muscles during the functional task.

The fundamental characteristic of this method of analysis to assess motor control capability is the use of the 
distribution of activity across the studied muscles during specific voluntary motor tasks [4]. VRI analysis is derived from the magnitude of the response vector, which is an expression of the overall activity or energy expended through the movement, and the similarity index as a result of the comparison of sEMG patterns recorded during voluntary movements to an average prototype pattern obtained from normal subjects. For example, a quantitative analysis of VRI was used from sEMG data recorded during defined voluntary movement to study changes in the magnitude and similarity muscle control pattern in subjects with incomplete spinal cord injury and it was reported that qualitative changes in sEMG patterns of motor control could be reflected by VRI [4].

The VRI of sEMG data had also been used to examine changes in the movement pattern of the cervical muscle groups during head reposition in young adults with chronic neck pain, and it was found that patients with chronic neck pain exhibited altered EMG patterns during voluntary sagittal neck motions [11]. The examples from these studies and several others indicated that VRI analysis could show changes in the CNS motor output that occurred with rehabilitative interventions or disorder progression [9, 11-13, 17].

The results of the current study showed high to very high levels of reliability ( $\mathrm{ICC}=0.75-0.92$ ) for MAG and SI. Although no available studies had assessed the reliability of VRI analysis in ACLR and healthy athletes in the mentioned functional task (sit-stand-sit), there was only one study that assessed the reliability of VRI analysis, and it was in subjects with incomplete Spinal Cord Injury (SCI) [18]. The results from that study (ICC $=0.65-0.95$ ) for MAG and SI may be comparable to the results in the current study with a difference in the applied protocol during motor tasks. They concluded that VRI analysis seemed to be a reliable method for shortand intermediate-term in subjects with SCI. Khaleghi et al. used VRI analysis to study the knee muscle reciprocal coactivation in patellofemoral pain syndrome; they reported an ICC of 0.85-0.99 for VRI components [12]. Furthermore, they found the lowest value of the absolute reliability of SI in both groups in all phases of the functional task. Moreover, the SI in both groups in all phases of the functional task had the lowest MDC. Lower scores of MDC of the VRI components suggested a sensitive amount of the measurement error [16].

In the current study, the reliability was evaluated in ACLR subjects. The study results are only valid for this population and not applicable to any other population. Thus, further research with a greater population is rec- ommended to evaluate this analysis method for other musculoskeletal disorders.

\section{Conclusion}

The current study showed a high to very high shortterm reliability of surface electromyographic measurements of VRI analysis in ACLR and healthy subjects during the functional motor task (sit-stand-sit). Thereby, it might be clinically appreciable to include this method of analysis to evaluate motor control in the protocols for examining the effects of rehabilitative interventions in subjects following an ACL reconstruction.

\section{Ethical Considerations}

\section{Compliance with ethical guidelines}

The voluntary participation of respondents in the research was considered. Moreover, participants had the right to withdraw from the study at any stage if they wished to do so. Full consent was obtained from the participants before the study. The anonymity of individuals participating in the research was ensured. The research did not have any emotional, physical, and financial harm to the participants. This research was approved by the Ethics Committee of Tehran University of Medical Sciences. (Ethical approval ID: IR.TUMS. VCR.REC.1398.571).

\section{Funding}

This research did not receive any specific grant from funding agencies in the public, commercial, or not-profit sectors.

\section{Authors contributions}

All authors contributed to preparing this paper.

\section{Conflict of interest}

The authors declared no conflict of interest.

\section{Acknowledgements}

We appreciate all the staff of the School of Rehabilitation who kindly helped us during the term of research. 


\section{References}

[1] Chhabra A, Starman JS, Ferretti M, Vidal AF, Zantop T, Fu FH. Anatomic, radiographic, biomechanical, and kinematic evaluation of the anterior cruciate ligament and its two functional bundles. The Journal of Bone and Joint Surgery. 2006; 88(Suppl. 4):2-10. [DOI:10.2106/JBJS.F.00616] [PMID]

[2] Fremerey RW, Lobenhoffer P, Zeichen J, Skutek M, Bosch $\mathrm{U}$, Tscherne $\mathrm{H}$. Proprioception after rehabilitation and reconstruction in knees with deficiency of the anterior cruciate ligament: A prospective, longitudinal study. The Journal of Bone and Joint Surgery. British Volume. 2000; 82(6):801-6. [DOI:10.1302/0301-620X.82B6.0820801] [PMID]

[3] Marx RG, Jones EC, Angel M, Wickiewicz TL, Warren RF. Beliefs and attitudes of members of the American Academy of Orthopaedic Surgeons regarding the treatment of anterior cruciate ligament injury. Arthroscopy: The Journal of Arthroscopic \& Related Surgery. 2003; 19(7):762-70. [DOI:10.1016/ S0749-8063(03)00398-0]

[4] Lee DC, Lim HK, McKay WB, Priebe MM, Holmes SA, Sherwood AM. Toward an objective interpretation of surface EMG patterns: a voluntary response index (VRI). Journal of Electromyography and Kinesiology. 2004; 14(3):379-88. . [DOI:10.1016/j.jelekin.2003.10.006] [PMID]

[5] Castanharo R, Da Luz BS, Bitar AC, D'Elia CO, Castropil W, Duarte M. Males still have limb asymmetries in multijoint movement tasks more than 2 years following anterior cruciate ligament reconstruction. Journal of Orthopaedic Science. 2011; 16(5):531-5. [DOI:10.1007/s00776-011-0118-3] [PMID]

[6] Lim HK, Lee DC, McKay WB, Protas EJ, Holmes SA, Priebe MM, Sherwood A. Analysis of sEMG during voluntary movement-part II: Voluntary response index sensitivity. IEEE Transactions on Neural Systems and Rehabilitation Engineering. 2004; 12(4):416-21.[DOI:10.1109/TNSRE.2004.838445] [PMID]

[7] Lim HK, Lee DC, McKay WB, Priebe MM, Holmes SA, Sherwood AM. Neurophysiological assessment of lower-limb voluntary control in incomplete spinal cord injury. Spinal Cord. 2005; 43(5):283-90. [DOI:10.1038/sj.sc.3101679] [PMID]

[8] Lin JJ, Lim HK, Soto-quijano DA, Hanten WP, Olson SL, Roddey TS, Sherwood AM. Altered patterns of muscle activation during performance of four functional tasks in patients with shoulder disorders: Interpretation from voluntary response index. Journal of Electromyography and Kinesiology. 2006; 16(5):458-68. [DOI:10.1016/j.jelekin.2005.09.008] [PMID]

[9] McKay WB, Ovechkin AV, Vitaz TW, De Paleville DT, Harkema SJ. Neurophysiological characterization of motor recovery in acute spinal cord injury. Spinal Cord. 2011; 49(3):421-9. [DOI:10.1038/sc.2010.145] [PMID] [PMCID]

[10] Zoghi M, Galea M, Morgan D. A Brain Motor Control Assessment (BMCA) protocol for upper limb function. PloS One. 2013; 8(11):e79483. [DOI:10.1371/journal. pone.0079483] [PMID] [PMCID]

[11] Cheng C-H, Wang J-L, Lin J-J, Wang S-F, Lin K-H. Position accuracy and electromyographic responses during head reposition in young adults with chronic neck pain. Journal of Electromyography and Kinesiology. 2010; 20(5):1014-20. [DOI:10.1016/j.jelekin.2009.11.002] [PMID]

[12] Khaleghi F, Olyaei GR, Talebian S, Malmir K, Bagheri H, Ansari NN, Jalaie S. Knee Muscle Reciprocal Co-Activation in Patellofemoral Pain Syndrome During Isokinetic Exer- cise: A Voluntary Response Index Analysis. Journal of Clinical Physiotherapy Research. 2016; 1(1):6-11. https://doi. org/10.22037/jcpr.v1i1.8733

[13] Norouzi Fashkhami A, Rahimi A, Kalantari KK. The voluntary response index in electromyographic study during landing test of the patients with ACL deficiency: A new study protocol. Iranian Red Crescent Medical Journal. 2014; 16(5):e14119. [DOI:10.5812/ircmj.14119] [PMID] [PMCID]

[14] Weir JP. Quantifying test-retest reliability using the intraclass correlation coefficient and the SEM. The Journal of Strength \& Conditioning Research. 2005; 19(1):231-40. [DOI:10.1519/15184.1] [PMID]

[15] Carter R, Lubinsky J, Domholdt E. Rehabilitation research principles and applications. $4^{\text {th }}$ edition. Philadelphia: Saunders; 2010. https://www.elsevier.com/books/rehabilitationresearch/carter/978-1-4377-0840-0

[16] Atkinson G, Nevill AM. Statistical methods for assessing measurement error (reliability) in variables relevant to sports medicine. Sports medicine. 1998; 26(4):217-38. [DOI:10.2165/00007256-199826040-00002] [PMID]

[17] McKay WB, Lee DC, Lim HK, Holmes SA, Sherwood AM Neurophysiological examination of the corticospinal system and voluntary motor control in motor-incomplete human spinal cord injury. Experimental Brain Research. 2005; 163(3):379-87. [DOI:10.1007/s00221-004-2190-9] [PMID]

[18] Lim HK, Sherwood AM. Reliability of surface electromyographic measurements from subjects with spinal cord injury during voluntary motor tasks. Journal of Rehabilitation Research \& Development. 2005; 42(4):413-22. [DOI:10.1682/ JRRD.2004.07.0079] [PMID] 